\title{
Endovascular Treatment of Intracranial Aneurysms Using the Novel Low Profile Visualized Intraluminal Support EVO Stent: Multicenter Early Feasibility Experience
}

\author{
Michelle Foo, MBBS (Hons) $)^{1}$, Julian Maingard, BBiomedSci, MBBS, FRANZCR ${ }^{2,3}$, Jonathan Hall, MBBS ${ }^{1,4}$, \\ Yifan Ren, MD $^{1}$, Goran Mitreski, BBiomed, MBBS ${ }^{1}$, Lee-Anne Slater, MBBS(Hons), FRANZCR, MMed, CCINR ${ }^{2,5}$, \\ Ronil Chandra, MBBS, MMed, FRANZCR, CCINR ${ }^{2,5}$, Winston Chong, MBBS, FRANZCR, CCINR ${ }^{2,6,7}$, \\ Ashu Jhamb, MBBS (Hons), FRANZCR, EBIR, CCINR ${ }^{1,4}$, Jeremy Russell, BSc/BE (Hons), MBBS (Hons), FRACS ${ }^{8}$, \\ Hong Kuan Kok, MB, BMedSci, MRCPI, MRCP (UK), FFR (RCSI), FRCR, EBIR ${ }^{3,9}$, \\ Mark Brooks, MBBS, FRANZCR, CCINR, EBIR ${ }^{1,4}$, Hamed Asadi, MD, PhD, FRANZCR, CCINR, EBIR ${ }^{1,2,3,10}$ \\ ${ }^{1}$ Interventional Neuroradiology Service, Department of Radiology, Austin Health, Heidelberg, VIC, Australia \\ ${ }^{2}$ Interventional Neuroradiology Service, Department of Radiology, Monash Health, Clayton, VIC, Australia \\ ${ }^{3}$ School of Medicine, Deakin University, Waurn Ponds, VIC, Australia \\ ${ }^{4}$ Interventional Neuroradiology Service, Department of Radiology, St Vincent's Hospital Melbourne, Fitzroy, VIC, Australia \\ ${ }^{5}$ Department of Imaging, Monash University, Clayton, VIC, Australia \\ ${ }^{6}$ Faculty of Medicine and Health Sciences, Macquarie University, Macquarie Park, NSW, Australia \\ ${ }^{7}$ Faculty of Medicine, Dentistry and Health Sciences, The University of Melbourne, Melbourne, VIC, Australia \\ ${ }^{8}$ Neurosurgery Department, Austin Health, Heidelberg, VIC, Australia \\ ${ }^{9}$ Interventional Radiology Service, Department of Radiology, Northern Health, Epping, VIC, Australia \\ ${ }^{10}$ Florey Institute of Neurosciences and Mental Health, The University of Melbourne, Parkville, VIC, Australia
}

Purpose: Low-profile, self-expandable stents have broadened therapeutic options available for definitive treatment of intracranial aneurysms. The novel Low-Profile Visualized Intraluminal Support (LVIS) EVO stent extends upon the success of its predecessor, the LVIS Jr stent, aiming to enable higher visibility and greater opening ability within a self-expandable and fully retrievable microstent system. In this study, we aim to report the early safety and feasibility experience with the LVIS EVO stent.

Materials and Methods: A multicenter, retrospective, observational study was conducted on patients who had intracranial aneurysms treated with the LVIS EVO stent across 3 Australian neurovascular centers between February 2020 and September 2020. Short-term technical and clinical outcomes were evaluated.

Results: A total of 22 LVIS EVO stents were successfully implanted to treat 15 aneurysms (3 ruptured, 12 unruptured) in 15 patients. Aneurysms ranged from $2 \mathrm{~mm}$ to $35 \mathrm{~mm}$ in dome height. The LVIS EVO stent was used for stent-assisted coiling in 11 patients and flow diversion in 4 patients. There were no device-related procedural complications. There were 2 cases of peri-procedural symptomatic thromboembolic complications and no procedure-related mortality. At early radiological follow up, 10 patients had complete occlusion, 4 patients had small neck remnants, and 1 patient who was managed with flow diversion had a residual aneurysm.

Conclusion: Early experience with the LVIS EVO stent demonstrated safety and feasibility for stent-assisted coiling as well as flow diversion for intracranial aneurysms. In this heterogeneous cohort, including ruptured, complex, and large aneurysms, all cases were technically successful.

Key Words: Intracranial aneurysm; Endovascular techniques; Interventional radiology instrumentation; Neuroimaging

\section{Correspondence to: Michelle Foo, MBBS (Hons) Interventional Neuroradiology Service, Department of Radiology, Austin Health, 145 Studley Rd., Heidelberg, VIC 3084, Australia Tel: +61-3-9496-5000 \\ E-mail: michelle.foo.10@gmail.com}

Received: April 17, 2021

Revised: May 23, 2021

Accepted: May 31, 2021

Copyright $\odot 2021$ Korean Society of Interventional Neuroradiology This is an Open Access article distributed under the terms of the Creative Commons Attribution Non-Commercial License (http://creativecommons.org/licenses/by-nc/4.0) which permits unrestricted non-commercial use, distribution, and reproduction in any medium, provided the original work is properly cited. 


\section{INTRODUCTION}

Stent-assisted coiling (SAC) is a well-established technique for the endovascular treatment of intracranial aneurysms, particularly those that are wide-necked. ${ }^{1}$ Among the diversified adjuvant stent technologies in recent years, the advent of low-profile, self-expandable stents has enabled compatibility with microcatheters down to internal diameters of 0.0165-inch, facilitating safer and easier navigation into distal or delicate arteries with diameters as small as $1.5 \mathrm{~mm}^{2}$ One such example is the Low-Profile Visualized Intraluminal Support (LVIS) and LVIS Jr stents (MicroVention, Tustin, CA, USA). After several clinical studies demonstrating long-term safety and efficacy for SAC of wide-necked aneurysms, these first-generation LVIS devices were the first stents to be granted premarket approval for intracranial SAC by the US Food and Drug Administration (FDA) in 2018, ahead of the Neuroform Atlas stent (Stryker, Kalamazoo, MI, USA) in 2019.3

The LVIS EVO device is the latest second-generation version of the LVIS Jr device which features improved visibility via drawn filled tube (DFT) wires, comprising a nitinol exterior and a platinum core (Fig. 1), alongside 4 radiopaque markers on each end of the implant and a $5 \mathrm{~mm}$ radiopaque pusher tip. It can be introduced by a microcatheter with an inner diameter of 0.0165 -inch or 0.017-inch. The flared ends of the LVIS EVO stent are shorter than predecessor LVIS stents (0.5 mm vs. $1.5-2.5 \mathrm{~mm}$ ), aiming to provide more flexible anchorage, particularly in small or tortuous vessels. The LVIS EVO stent is available in a variety of sizes, ranging from $2.5 \mathrm{~mm}$ to $4.0 \mathrm{~mm}$ in outer diameter, and from $12 \mathrm{~mm}$ to $34 \mathrm{~mm}$ in length. Once deployed, the stent can be deployed and resheathed by a single operator thus facilitating easier repositioning. A degree of flow-diversion is provided by its closedcell design with increased metal coverage (28\%) compared to previous intracranial stents such as the LVIS Jr (17-23\%), LEO (Balt Extrusion, Montmorency, France) (14\%), and Neuroform (5-10\%). ${ }^{3-5}$ This degree of metal coverage is approaching that of dedicated flow-diverting stents, which generally have at least 35\% metal coverage in order to achieve greater than 95\% angiographic aneurysm occlusion. ${ }^{6}$

Our study aims to report our early safety and feasibility experience with the LVIS EVO stent when used in SAC and flow-diversion (FD) applications for the treatment of ruptured and unruptured intracranial aneurysms.

\section{MATERIALS AND METHODS}

A multicenter retrospective observational study was conducted across 3 tertiary referral hospitals in Australia. This study included all consecutive patients with non-dissecting intracranial aneurysms treated endovascularly with the LVIS EVO stent over an 8-month period from 1st February 2020 to 30th September 2020. Six fellowship-trained neurointerventionists were involved as the primary operators across these cases. Data were retrospectively collected from the electronic medical records by investigators with pre-existing access to the records at each of the 3 institutions. This study was approved by the Institutional Review Boards.

\section{Medications}

All elective cases received dual antiplatelet pre-medication with acetylsalicylic acid (ASA; 100 mg daily) and either clopi-

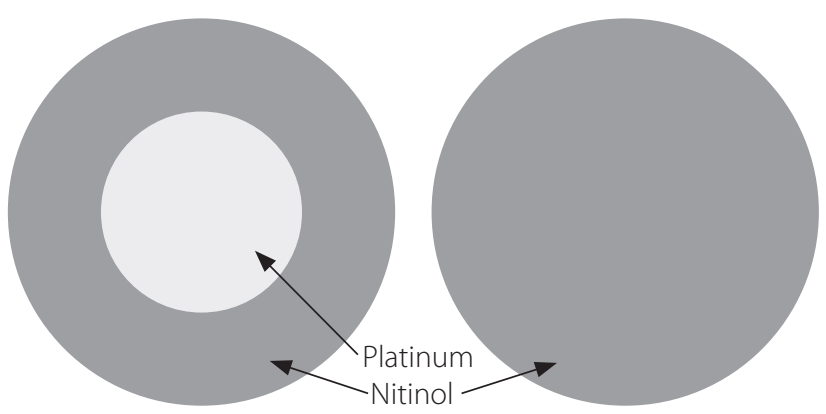

A

B

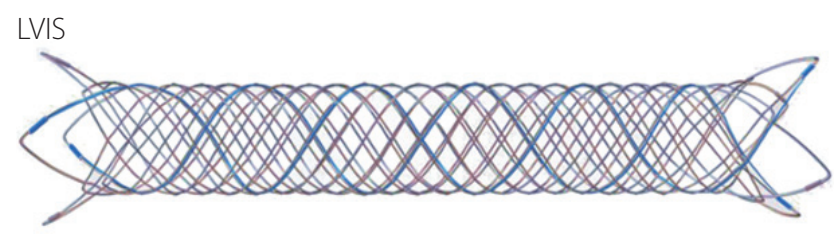

LVIS Jr

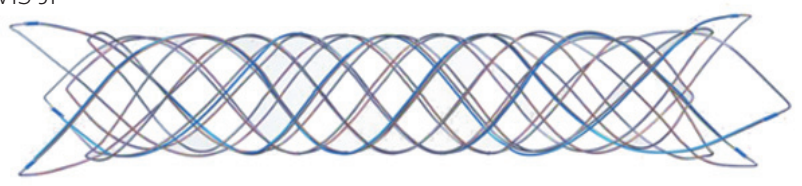

LVIS EVO

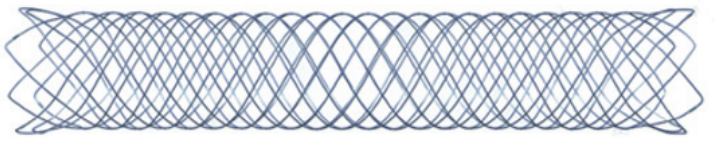

C

Fig. 1. Drawn-filled tube wire technology of the Low-Profile Visualized Intraluminal Support (LVIS) EVO device (A), allowing radio-opacity of the entire length of the stent body, as compared to the pure nitinol wires used in LVIS and LVIS Jr (B). (C) Visual comparison between LVIS, LVIS Jr, and LVIS EVO stents. 
dogrel (75 mg daily), prasugrel (10 mg daily), or ticagrelor (90 mg twice daily) for at least 5 days before the procedure. In emergency cases, antiplatelet pre-medication was given with $300 \mathrm{mg}$ clopidogrel via the oral or nasogastric route, as well as 1,000 mg of intravenous (IV) ASA administered th the commencement of the procedure. Intraprocedural IV heparin $(80 \mathrm{lU} / \mathrm{kg})$ was administered in all cases.

\section{Procedural technique}

All procedures were performed under general anesthesia in a dedicated biplane angiography suite. Common femoral artery access was obtained followed by insertion of an introducer sheath between 6F and 9F. In cases of SAC, a "semi-jailing" double microcatheter technique was employed. A first microcatheter was navigated into the aneurysm sac (Fig. 2A) before partial deployment of the LVIS EVO stent across the aneurysm neck (Fig. 2B) and subsequent delivery of coils via the first microcatheter (Fig. 2C). Whereas the traditional jailing technique is more compatible with the LVIS Jr stent, this technique is more suitable for the LVIS EVO stent because the microcatheter can be difficult to recross the high metal coverage walls in case microcatheter kick-back occurs. Additionally, partial deployment during coil-packing allows the first microcatheter a degree of mobility during coil delivery, so there is some control over the coil mass. Once

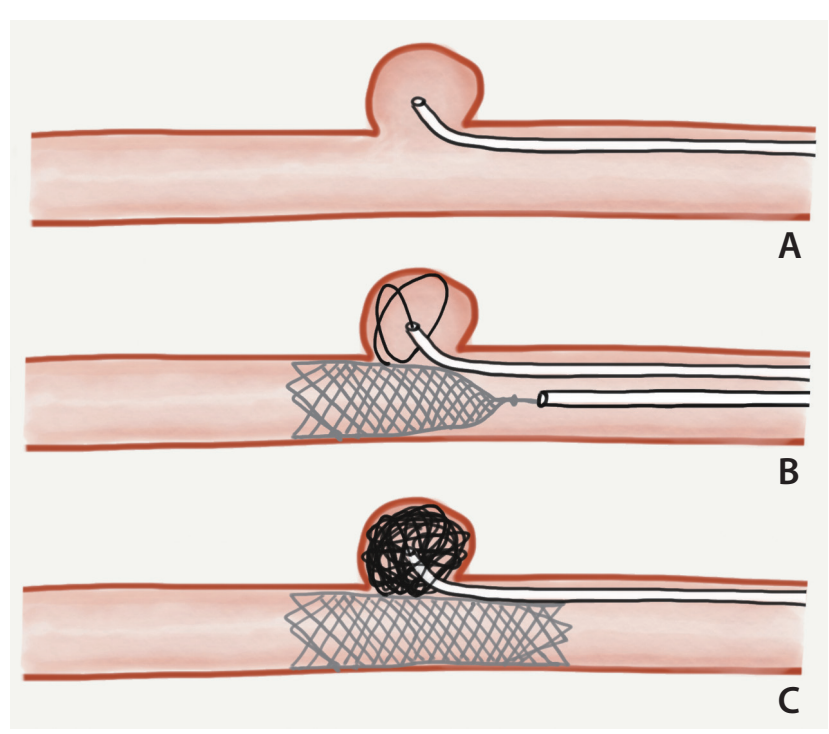

Fig. 2. Semi-jailing double microcatheter technique. (A) The first microcatheter is positioned with its tip in the aneurysm sac. (B) A second microcatheter is used to partially deploy the stent across the aneurysm neck, securing the first microcatheter between the stent and the vessel wall. (C) The stent is fully deployed after dense coil packing. dense coil packing is achieved, the stent is fully deployed and the final coil is delivered, preventing microcatheter "kickback" at this point.

For cases of SAC, immediate occlusion results were graded on the final digital subtraction angiography (DSA) run using the modified Raymond-Roy Occlusion Classification (RROC): complete occlusion (Class I); residual neck (Class II); residual aneurysm with contrast within coil interstices (Class IIla); or residual aneurysm with contrast along the aneurysmal wall (Class IIIb). ${ }^{7}$ For cases of FD, immediate angiographic outcomes were graded using the O'Kelly-Marotta (OKM) scale, in which the initial degree of filling is graded in descending $\operatorname{order}(A, B, C, D)$ and the degree of stasis is graded in ascending order $(1,2,3){ }^{8}$

\section{Post-procedural care and follow-up}

Post-procedurally, patients were transferred to a high dependency unit or intensive care unit for strict hemodynamic and neurological monitoring. All patients commenced dual antiplatelet therapy (the same 2 agents given pre-procedurally, or if given IV ASA loading, aspirin and clopidogrel) from 24 hours post-procedure, for at least 6 months. Elective patients were discharged after at least 24 hours of post-procedural monitoring. For patients treated emergently, non-contrast brain computed tomography was performed day 1 post-procedure to assess for change in pathology and screen for procedural complications, such as infarction or hemorrhage. All patients were planned to undergo imaging follow-up within 1 to 3 months post-procedure with brain magnetic resonance imaging (MRI) including 3D time of flight (TOF) magnetic resonance angiography (MRA) (MRI/MRA).

\section{RESULTS}

A total of 15 intracranial aneurysms in 15 patients were treated using 22 LVIS EVO stents across 3 tertiary referral centers over the study period (between February-September 2020). The study population comprised 10 females and 5 males, with a mean age of 63 years (range: $49-90$ years). All except 2 aneurysms were considered wide-necked, as per the following consensus definition: neck width $\geq 4 \mathrm{~mm}$ or dome width-to-neck width ratio of $<2 .{ }^{9}$ Further details about each patient's presentation, aneurysm characteristics, procedural technique, and outcomes are summarized in Table 1.

Three of the aneurysms were ruptured; 2 of which were 


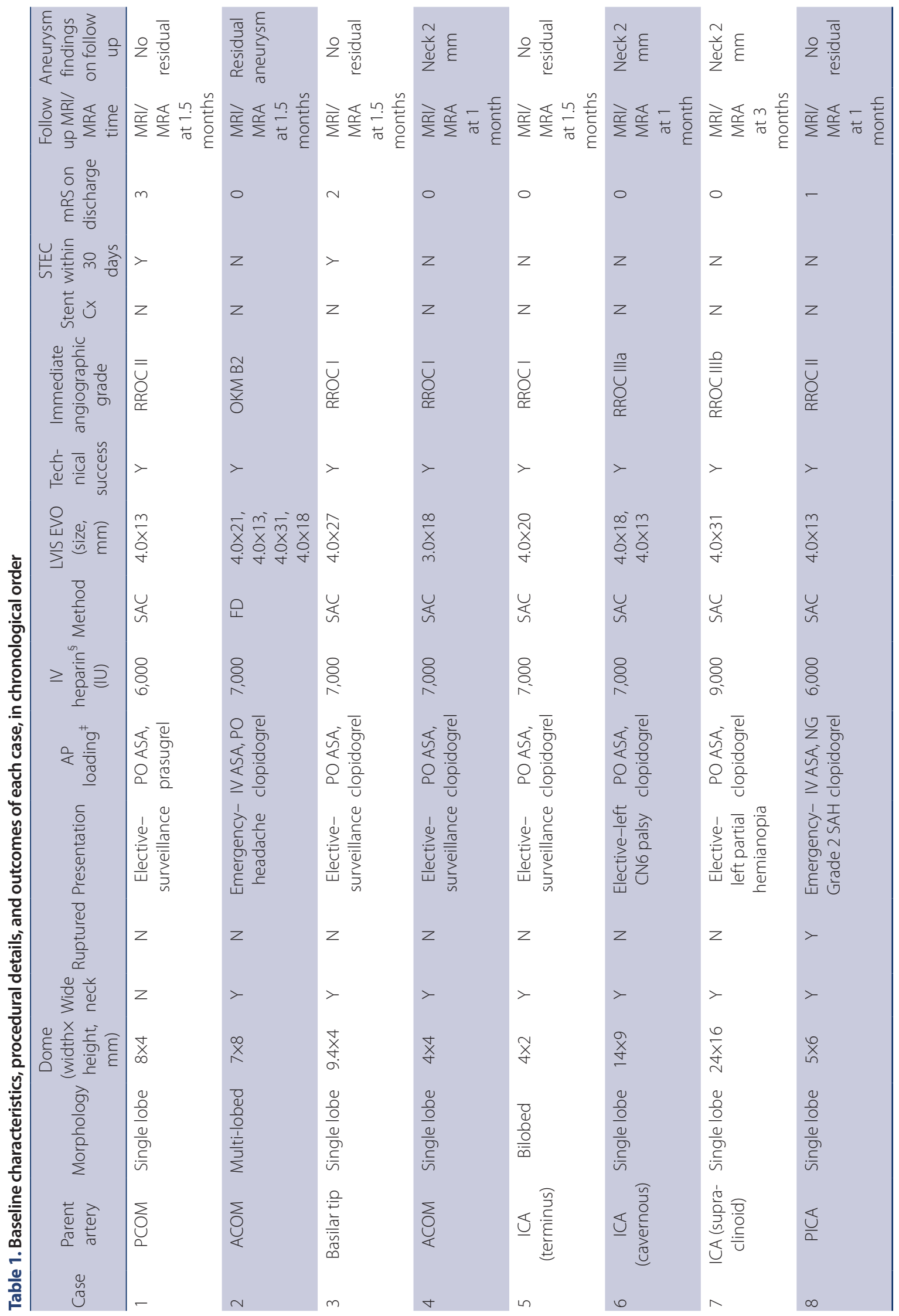




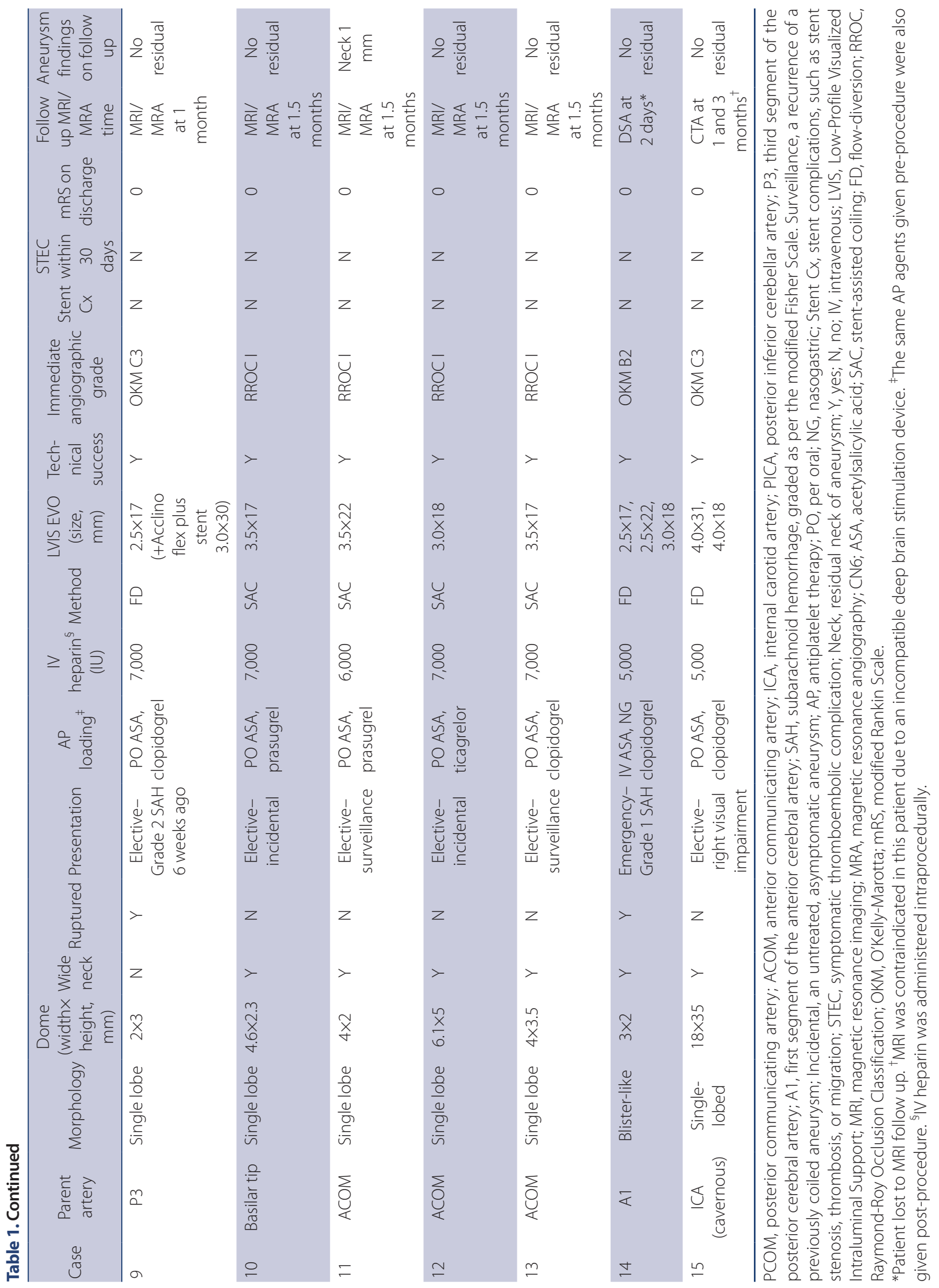


readily identified as the source of subarachnoid hemorrhage $(\mathrm{SAH})$ and hence treated emergently (Case 8 and 14). The remaining ruptured aneurysm (Case 9, Fig. 3) was only identified once thrombosed on a delayed MRI performed 6 weeks post-SAH; hence the patient was readmitted for elective stent-assisted coiling. Of the 12 patients with unruptured aneurysms, all were treated electively except for 1 patient (Case 2) who presented to the emergency department with a thunderclap headache and underwent urgent endovascular treatment of a complex multilobular aneurysm.

Eleven patients had their aneurysm coiled with assistance from the LVIS EVO stent to stabilize the coils and prevent coil prolapse. In the remaining 4 patients (Cases 2, 9, 14, and 15), the LVIS EVO device was used for flow diversion. The reasons for using flow diversion were varied. Case 2 presented with high clinical suspicion for SAH and in an emergent investigative DSA, 4 LVIS EVO stents were deployed into the anterior communicating artery (ACOM), left $A 1$, and left $A 2$ segments to exclude a $7 \times 8 \mathrm{~mm}$ (dome width $\times$ height) multilobulated aneurysm with a high rupture risk. Its location at the left ACOM-ACA bifurcation meant that coil embolization posed an unacceptable risk of coil protrusion, and a small recurrent artery of Heubner arising from the aneurysmal sac meant that coil embolization also presented a risk of iatrogenic Heubner cerebral infarction. Case 9 was also treated with FD due to the dysplastic morphology and a small caliber of the parent posterior cerebral artery (PCA) (P3 segment), prohibiting access for 2 catheters. Case 14 was treated with FD given the small blister-like aneurysm, which implied that SAC would present a higher risk of rupture due to thinner aneu- rysmal walls. ${ }^{10}$ For Case 15 , FD was pursued because of the giant size of the cavernous aneurysm (18×35 mm). Two LVIS EVO stents were specifically selected for this case, aiming to utilize the flexibility and opening ability of the stent in order to accommodate the size mismatch between the proximal and distal portions of the stenosed internal carotid artery (ICA).

All cases had a technically successful result, with no failed deployments and no intraprocedural complications. Of the 11 patients treated with SAC, 9 patients achieved adequate occlusion (RROC I in 7 patients and RROC II in 2 patients). The remaining 2 patients (Cases 6 and 7) had large aneurysms (dome width of $14 \mathrm{~mm}$ and $24 \mathrm{~mm}$ ), where RROC IIla and Illb occlusion was achieved respectively and on short-term follow-up MRI/MRA, and both had a minor residual aneurysm neck (Fig. 4). This was expected given that a calculated decision was made to avoid dense coil packing in order to prevent long-term mass effect of the coiled aneurysm onto the parent artery and to allow for gradual thrombosis via flow-diverting properties of the stent.

For all 4 patients treated with FD, satisfactory stent positioning was achieved with good aneurysm neck coverage. On the immediate post-treatment angiogram, all patients had reduced aneurysmal filling and increased stasis, with 2 cases achieving OKM grade B2 and 2 achieving OKM grade C3.

In the 30-day post-procedure period, there were no deaths. Two patients (13.3\%) experienced symptomatic thromboembolic complications (Cases 1 and 3) and were discharged with modified Rankin Scale (mRS) scores of 3 and 2 respectively. Case 1 was an elderly patient who underwent elective
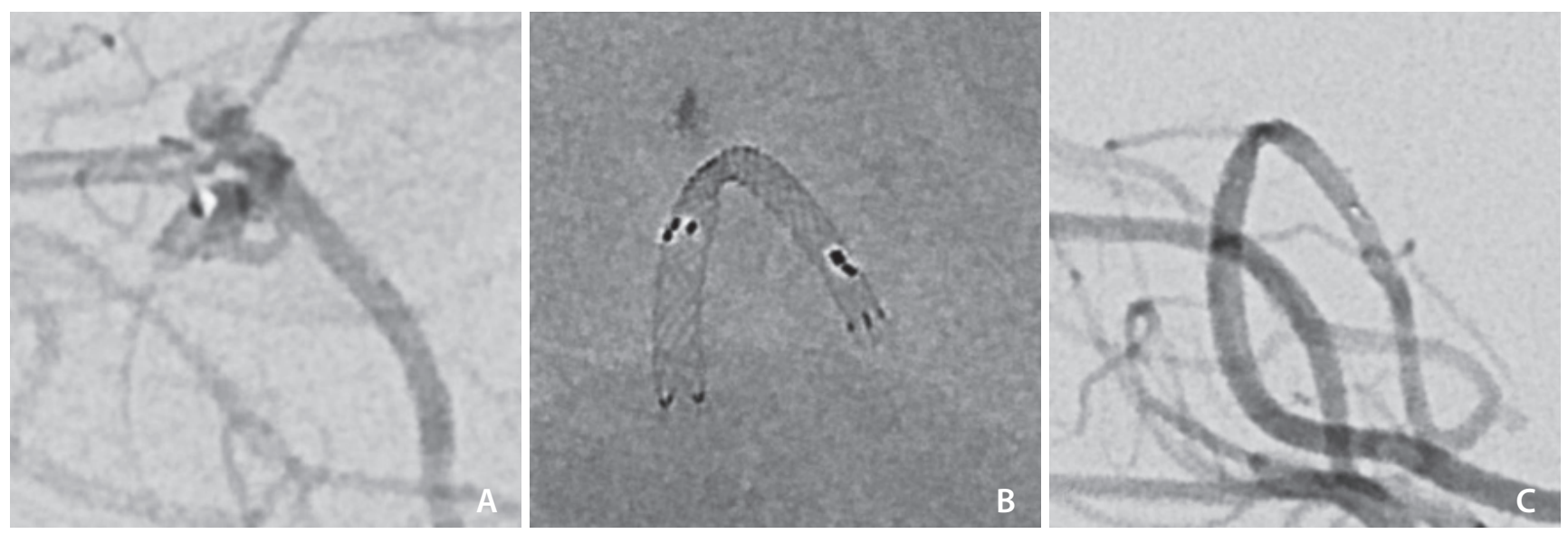

Fig. 3. Case 9. (A) Microcatheter tip near the base of a small aneurysm arising from a tortuous right P3 segment. (B) The Low-Profile Visualized Intraluminal Support (LVIS) EVO stent was easily and successfully deployed as a flow-diverting stent within an Acclino flex plus stent, achieving significant slowing of inflow into the aneurysmal sac on final angiographic run (C). 
SAC of an unruptured recurrent posterior communicating artery aneurysm. Having received positive pressure ventilation, the patient unfortunately suffered a significant haemopneumothorax during the procedure and thus required emergent IV platelets and heparin reversal. Along with a period of hypotension, this likely contributed to the thromboembolic complication, a small cortical right superolateral frontal lobe infarction. Case 3 was a patient with a recurrent basilar tip aneurysm after previous rupture and treatment with a WEB device (Sequent Medical Inc., Aliso Viejo, CA, USA). One day after an uncomplicated elective SAC, achieving RROC I, the patient developed ataxia and hemianopia and was found to have a left paramedian midbrain infarct, which was managed conservatively. This patient was discharged home and on 6-week clinical follow-up had an mRS score that improved from 2 to 1 , with an ongoing degree of right visual field loss only. One other patient was discharged with an mRS score of 1-an elderly patient with acute SAH (Case 8), who was discharged to rehabilitation in a generally deconditioned state without focal neurological deficits. The remaining 12 patients (80.0\%) were discharged home with an $\mathrm{mRS}$ score of 0 .

In terms of radiological follow-up, 13 patients (86.7\%) received an MRI/MRA within 1-3 months (average 1.5 months). Of the 2 patients who did not undergo follow-up MRI/MRA, 1 (Case 15) had an absolute contraindication to MRI so underwent CT angiography (CTA) instead at 1 and 3 months, while the other patient was lost to MRI/MRA follow-up.

At follow-up MRI, 8 patients (61.5\%) had complete occlusion of the aneurysm, while 4 patients (30.8\%) had a minor neck remnant and 1 patient (7.7\%) had residual aneurysm after FD (Case 2). In Case 2, given that the flow signal was
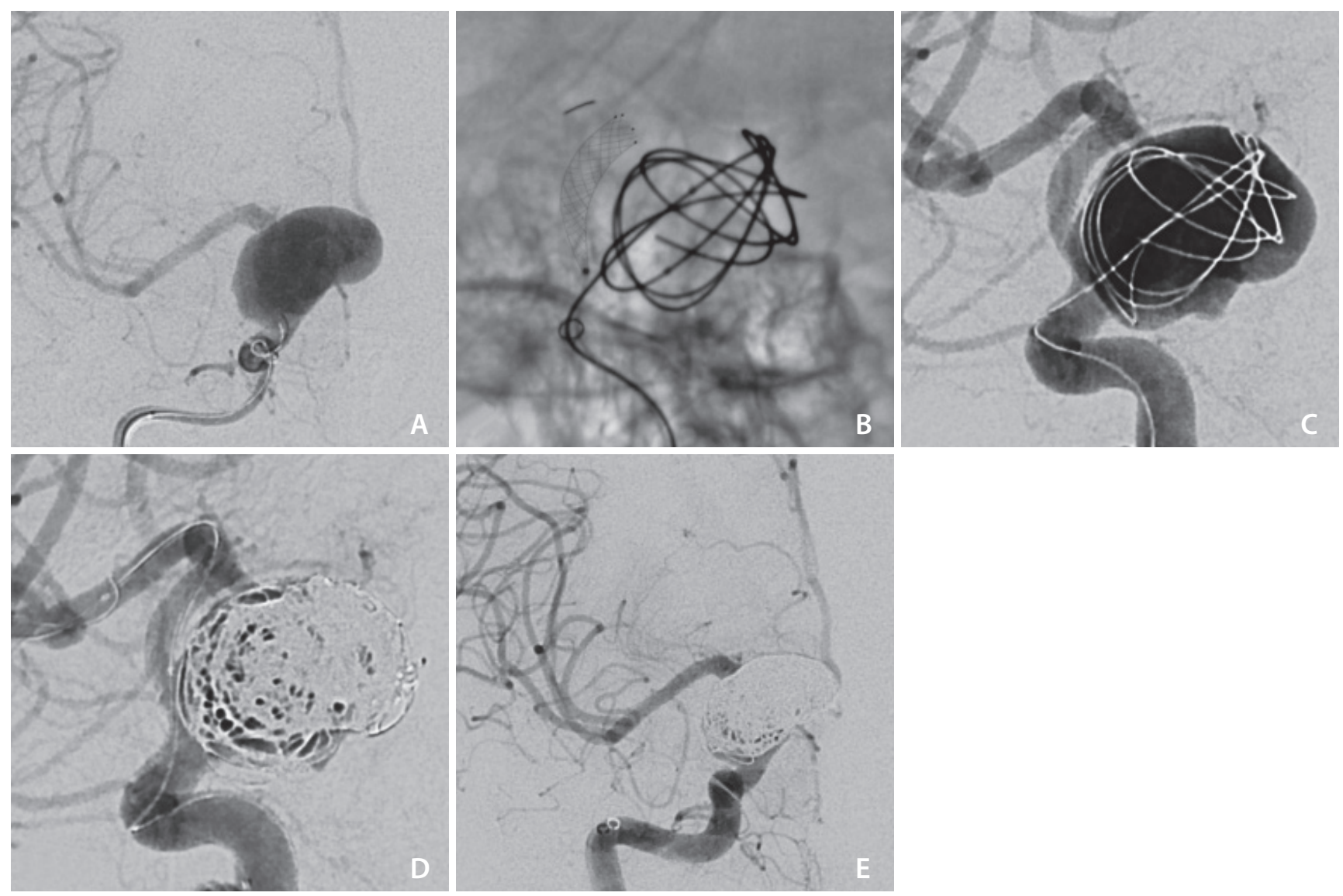

Fig. 4. Low-Profile Visualized Intraluminal Support (LVIS) EVO-assisted coiling of an unruptured right supraclinoid internal carotid artery (ICA) aneurysm. (A) Frontal projection DSA with contrast injection in the right cavernous ICA. (B) LVIS EVO device amid deployment, with radiopaque delivery tip visible within the right MCA and 4 radiopaque markers at the distal end of the implant. Note undetached coils within the aneurysm sac to help with stability of the jailed microcatheter. (C, D) Progressive packing of the aneurysm with coils. (E) Final DSA run demonstrating a subtle degree of contrast opacification along the aneurysmal wall. 
reduced compared to baseline imaging, and factoring in the aforementioned complex anatomy of the aneurysm which rendered treatment technically challenging, it was deemed an acceptable early outcome and a multidisciplinary decision was made to step down to single-agent antiplatelet therapy to facilitate gradual aneurysm thrombosis.

\section{DISCUSSION}

The latest generation LVIS EVO stent has extended upon the success of its predecessors in broadening the scope for endovascular treatment of aneurysms associated with widenecked, tortuous, or delicate vascular anatomy.

Our results suggest that DFT technology results in improved visibility of the stent under fluoroscopy allowing more controlled stent positioning. There was a 100\% success rate in LVIS EVO stent implantations in our series with complete neck coverage and good wall apposition with no cases requiring repositioning. Despite the LVIS EVO stent featuring shorter flared ends than its predecessors, this did not affect stent anchorage upon deployment and there were no cases of stent migration intra-procedurally or on follow-up angiography.

Furthermore, the improved radial force was evidenced by neither coil prolapse nor ribboning, as well as robust opening ability even in complex vascular anatomy. For example, in the tortuous and stenosed P3 segment of Case 9, it was possible to navigate and deliver the LVIS EVO stent system to the aneurysm easily. In this case, a $2.5 \times 17 \mathrm{~mm}$ LVIS EVO stent was deployed without difficulty within a $3.0 \times 30 \mathrm{~mm}$ Acclino flex plus stent (Acandis, Pforzheim, Germany) to provide more radial force against the tightly stenosed artery (1.5 mm diameter), achieving immediate slowing of inflow and stasis within the aneurysmal sac. In this case, the stent's braided wires with small cell size provided an excellent FD effect within the small artery. Additionally, despite the increased risk of stent thrombosis associated with higher metal coverage, all patients with early angiographic follow-up had patent stents.

While the rate of complete occlusion (RROC I) on the final DSA run was only $63.6 \%(7 / 11)$ in the SAC population, 3 out of the 4 incompletely coiled aneurysms included 2 large, widenecked aneurysms (Case 6 and 7) which deemed to be at high risk for coil protrusion, and 1 acutely ruptured aneurysm (Case 8). These cases had no periprocedural complications and demonstrated satisfactory results on MRA follow-up.

The 2 cases of symptomatic thromboembolic complication were unexpected in the context of both being elective procedures in unruptured aneurysms with planned preprocedural antiplatelet loading and no intraprocedural difficulties. In one of these, the intraprocedural haemopneumothorax, possibly related to positive pressure ventilation, led to hypotension and hypovolaemia requiring platelet and whole blood transfusion, which would have contributed to a prothrombotic state, increasing embolic risk. In the other case, it is possible that the patient may have had a suboptimal response to aspirin or clopidogrel, given that platelet activity tests are not routinely performed at our institutions. These 2 cases suggest that the complication rate from elective treatment was relatively high at 17\% (2/12); however, this is biased by a small sample size, and the complications are not invariably related to the properties of the stent nor techniques associated with its usage.

In comparison to our study with immediate complete occlusion (RROC 1) in 64\% (7/11) of SAC cases, 2 recent single-center observational studies of LVIS EVO SAC (Sirakov et al. ${ }^{11}$ [n=6]; Poncyljusz et al. ${ }^{12}$ [n=35]) both reported immediate RROC 1 in 100\% of cases. The poorer angiographic outcomes in our study may be explained by a number of differences in baseline aneurysm characteristics. First, we included a greater proportion of patients with posterior circulation aneurysms than Sirakov's or Poncyljusz's studies (38.5\% [5/13] vs. $16.7 \%[1 / 6]$ vs. $11.4 \%[4 / 35])$. It is established that endovascular treatment of posterior circulation aneurysms is technically more challenging and carries a higher risk of recurrence than that of anterior circulation aneurysms. ${ }^{12,13}$ Second, our series featured large and complex aneurysms, compared to Sirakov's study, which only included small saccular aneurysms ( $\leq 10 \mathrm{~mm}$ in dome height) that are less predisposed to recurrence. ${ }^{14}$ In comparison to Poncyljusz's study, our series featured a greater proportion of wide-necked aneurysms (45.7\% [16/35] vs. 86.7\% [13/15]), in which complete occlusion is technically more challenging to achieve. Finally, a greater proportion of our cases were treated in an emergency setting (26.7\% [4/15]) compared to Sirakov's study, which only included elective, unruptured, or previously treated aneurysms, as well as Poncyljusz's study, which had $17.1 \%$ (6/35) of emergency cases. Compared to elective settings, emergency aneurysm treatment is generally challenged by greater intraand peri-procedural risks and less opportunity for preprocedural planning. ${ }^{14}$ 
The third and largest study to date reporting the use of the LVIS EVO stent is Vollherbst's recent retrospective multicenter review of 57 patients across 11 European neurovascular centers. ${ }^{15}$ Similar to our study, Vollherbst's study population included several complex patient and aneurysm characteristics, such as emergency cases (15\% SAH, 3.4\% ischemic stroke), non-saccular aneurysms (5.1\% dissecting aneurysms, $1.7 \%$ blisterlike aneurysms), and wide-necked aneurysms (95\%). The rate of immediate complete occlusion was 54\%, as compared with 64\% (7/11) in our SAC cases, and 100\% in Sirakov and Poncyljusz's studies-again suggesting the effect of patient selection. Of note, Vollherbst's study is the first to report any cases of failed LVIS EVO stent deployment (6.8\% [ $\mathrm{n}=4]$ required in-stent angioplasty) or periprocedural stent-related complications (10.2\% [n=6]). These complications included 3 cases of in-stent thrombosis, 1 case of coil protrusion into the parent vessel, 1 case of stent shortening, and 1 case of insufficient opening. Two of the cases of instent thrombosis may be explained by the fact they were ruptured aneurysms where pre-procedure antiplatelet loading was prohibited. Furthermore, the case of insufficient stent opening occurred in a tortuous ICA $\left(180^{\circ}\right.$ curve at proximal stent site), while the case of stent shortening occurred only after in-stent angioplasty. Given that Vollherbst's study is the first published report of difficulties opening the LVIS EVO stent, as well as shortening with subsequent balloon remodeling, further studies with technical data are of value in order to delineate risk factors for unsuccessful deployment.

As an early feasibility study, this study has several limitations. There is a high risk of sampling bias given the small patient population examined. Additionally, the heterogeneity of the patient population, such as the inclusion of ruptured aneurysms, introduces confounding factors that may substantially skew outcomes but are also more reflective of real-world practice in major neurovascular centers. In-person follow-up was prohibited for most patients in the context of local restrictions enforced during the COVID-19 pandemic, and hence early clinical follow-up was limited in this study. Moreover, the short imaging follow-up period precludes accurate assessment of medium-to-long term outcomes and the need for re-intervention.

\section{CONCLUSION}

The present multicenter study is the first Australian study to date describing the use of the LVIS EVO device in clinical practice for the treatment of intracranial aneurysms. Across a range of elective and emergency situations, the LVIS EVO device demonstrated feasibility and safety for both SAC and FD techniques. Prospective, comparative, larger-scale studies are necessary to confirm the long-term efficacy, safety, and potential superiority of the LVIS EVO stent relative to other existing devices.

\section{Fund}

None.

\section{Ethics Statement}

This study was approved by the local Institutional Review Board and the need for written informed consent was waived.

Since the consent for publication was not available in this report, the patients' information was anonymized by removing the sex and specific age.

\section{Conflicts of Interest}

The authors have no conflicts to disclose.

\section{Author Contributions}

Concept and design: MB and HA. Analysis and interpretation: MF. Data collection: MF, JM, JH, and YR. Writing the article: MF, $J M$, and HKK. Critical revision of the article: GM, LS, RC, WC, $A J, J R$, and HKK. Final approval of the article: HA. Statistical analysis: MF. Overall responsibility: MF.

\section{ORCID}

Michelle Foo: https://orcid.org/0000-0001-8056-1163 Julian Maingard: https://orcid.org/0000-0001-8958-2411 Jonathan Hall: https://orcid.org/0000-0002-5952-2992 Yifan Ren: https://orcid.org/0000-0001-6518-6828 Goran Mitreski: https://orcid.org/0000-0003-0312-0617 Lee-Anne Slater: https://orcid.org/0000-0002-1140-8664 Ronil Chandra: https://orcid.org/0000-0001-7555-2297 Winston Chong: https://orcid.org/0000-0001-7645-2861 Ashu Jhamb: https://orcid.org/0000-0002-7097-1641 Jeremy Russell: https://orcid.org/0000-0003-3692-120X Hong Kuan Kok: https://orcid.org/0000-0002-8740-6067 Mark Brooks: https://orcid.org/0000-0001-5034-570X Hamed Asadi: https://orcid.org/0000-0003-2475-9727 


\section{REFERENCES}

1. Phan K, Huo YR, Jia F, Phan S, Rao PJ, Mobbs RJ, et al. Meta-analysis of stent-assisted coiling versus coiling-only for the treatment of intracranial aneurysms. J Clin Neurosci 2016;31:15-22

2. Aydin K, Arat A, Sencer S, Barburoglu M, Men S. Stent-assisted coiling of wide-neck intracranial aneurysms using low-profile LEO baby stents: initial and midterm results. AJNR Am J Neuroradiol 2015;36:1934-1941

3. Fiorella D, Boulos A, Turk AS, Siddiqui AH, Arthur AS, Diaz O, et al.; LVIS investigators. The safety and effectiveness of the LVIS stent system for the treatment of wide-necked cerebral aneurysms: final results of the pivotal US LVIS trial. J Neurointerv Surg 2019;11:357-361

4. Wang C, Tian Z, Liu J, Jing L, Paliwal N, Wang S, et al. Flow diverter effect of LVIS stent on cerebral aneurysm hemodynamics: a comparison with Enterprise stents and the Pipeline device. J Trans/ Med 2016;14:199

5. Cagnazzo F, Cappucci M, Dargazanli C, Lefevre PH, Gascou G, Riquelme C, et al. Flow-diversion effect of LEO stents: aneurysm occlusion and flow remodeling of covered side branches and perforators. AJNR Am J Neuroradio/ 2018;39:2057-2063

6. Wang K, Huang Q, Hong B, Li Z, Fang X, Liu J. Correlation of aneurysm occlusion with actual metal coverage at neck after implantation of flow-diverting stent in rabbit models. Neuroradiology 2012;54:607-613

7. Mascitelli JR, Moyle H, Oermann EK, Polykarpou MF, Patel AA, Doshi AH, et al. An update to the Raymond-Roy Occlusion Classification of intracranial aneurysms treated with coil embolization. J Neurointerv Surg 2015;7:496-502

8. O'kelly CJ, Krings T, Fiorella D, Marotta TR. A novel grading scale for the angiographic assessment of intracranial aneurysms treated using flow diverting stents. Interv Neuroradiol 2010;16:133-137

9. Hendricks BK, Yoon JS, Yaeger K, Kellner CP, Mocco J, De Leacy RA, et al. Wide-neck aneurysms: systematic review of the neurosurgical literature with a focus on definition and clinical implications. [published online ahead of print Jun 14, 2019] J Neurosurg 2019

10. Regelsberger J, Matschke J, Grzyska U, Ries T, Fiehler J, Köppen J, et al. Blister-like aneurysms--a diagnostic and therapeutic challenge. Neurosurg Rev 2011;34:409-416

11. Sirakov A, Bhogal P, Möhlenbruch M, Sirakov S. Endovascular treatment of patients with intracranial aneurysms: feasibility and successful employment of a new low profile visible intraluminal support (LVIS) EVO stent. Neuroradiol J 2020;33:377-385

12. Poncyljusz W, Kubiak K. Initial experience with LVIS EVO stents for the treatment of intracranial aneurysms. J Clin Med 2020;9:3966

13. Pandey AS, Koebbe C, Rosenwasser RH, Veznedaroglu E. Endovascular coil embolization of ruptured and unruptured posterior circulation aneurysms: review of a 10-year experience. Neurosurgery 2007;60:626-636; discussion 636-637

14. Ferns SP, Sprengers ME, van Rooij WJ, Rinkel GJ, van Rijn JC, Bipat S, et al. Coiling of intracranial aneurysms: a systematic review on initial occlusion and reopening and retreatment rates. Stroke 2009:40:e523-e529

15. Vollherbst DF, Berlis A, Maurer C, Behrens L, Sirakov S, Sirakov A, et al. Periprocedural safety and feasibility of the new LVIS EVO device for stent-assisted coiling of intracranial aneurysms: an observational multicenter study. AJNR Am J Neuroradiol 2021;42:319-326 